\title{
The influence of symmetry on the conformational dependences of various molecular parameters
}

Cite as: J. Chem. Phys. 63, 4515 (1975); https://doi.org/10.1063/1.431131

Published Online: 03 September 2008

J. Maruani, A. Hernandez-Laguna, and Y. G. Smeyers

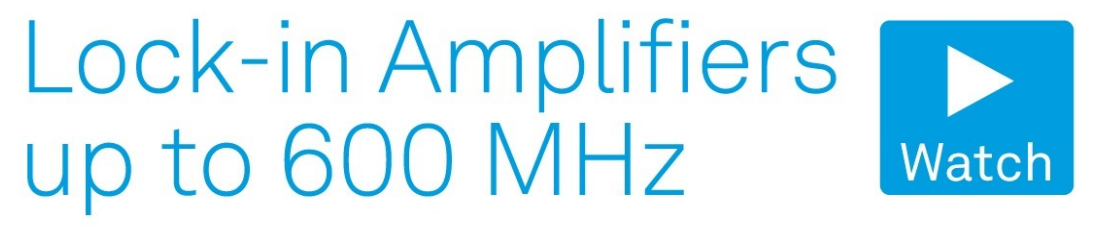




\title{
The influence of symmetry on the conformational dependences of various molecular parameters
}

\author{
J. Maruani, ${ }^{*}$ A. Hernandez-Laguna, ${ }^{\dagger}$ and Y. G. Smeyers ${ }^{\dagger}$ \\ Centre de Mécanique Ondulatoire Appliquée, Centre National de la Recherche Scientifique, 23, Rue du \\ Maroc, Paris-19, France \\ (Received 18 June 1975) \\ It is shown how the symmetry properties of the linked groups in a molecule help to reduce the number of \\ harmonics in the Fourier series expansions of aggregate, mononuclear, and binuclear parameters as a \\ function of the dihedral angles. This provides a rationalization for some well-known conformational \\ dependences of the torsional potentials, hyperfine couplings, and internuclear interactions and helps to \\ predict how these simple formulas break down when the conformations become more complex.
}

\section{INTRODUCTION}

Among the $3 N-6$ internal degrees of freedom of an $N$-atom nonlinear molecule, the $n \leqslant N-3$ torsional dihedral angles usually involve particularly low energy barriers when the atoms lying on the internal-rotation axis are single bonded. ${ }^{1}$ A full relative reorientation of the bonded groups around the bond axis can then be performed without bringing about very strong changes in the remaining features of the nuclear configuration and electronic distribution of the molecule. As a consequence, the dependence of most molecular parameters on the torsional dihedral angles can reasonably be described with the first few significant harmonics of an appropriate Fourier series expansion. Symmetry considerations can sometimes lead to further reduction in the number of harmonics necessary to represent the conformational dependence of a defined parameter in a given molecule. The purpose of the present paper is to derive some practical rules for obtaining simple expressions of various molecular parameters as a function of conformational angles.

\section{THEORY AND DISCUSSION}

Let us consider a molecule consisting of a reference molecular group and of $n$ rotors defining the $n$ torsional dihedral angles $\theta_{i}$. In general, the dependence of any parameter $A$ on one angle $\theta_{i}$ can be written as

$$
\begin{aligned}
A\left(\theta_{i}\right) & =\sum_{i=0}^{\infty}\left(A_{i 1}^{c} \cos 1 \theta_{i}+A_{i 1}^{s} \sin 1 \theta_{i}\right) \\
& \equiv \sum_{i=0}^{\infty} A_{i 1} \cos 1\left(\theta_{i}-\phi_{i 1}\right) \equiv \sum_{i=-\infty}^{+\infty} \tilde{A}_{i 1} \exp i 1 \theta_{i},
\end{aligned}
$$

where the $A_{i 1}$ 's and $\phi_{i 1}$ 's are periodic functions of other $\theta$ 's. If the $n \theta$ 's are simultaneously changed, $A$ can be developed on a basis which is a tensorial product of bases similar to the previous one, that is,

$$
\begin{aligned}
& A\left(\theta_{1}, \ldots, \theta_{n}\right)=\sum_{1_{1}=0}^{\infty} \cdots \sum_{1_{n}=0}^{\infty}\left(A_{1_{1} \ldots 1_{n}}^{c \ldots c} \cos 1_{1} \theta_{1} \ldots \cos 1_{n} \theta_{n}+A_{1_{1} \ldots 1_{n}}^{c \ldots . s s} \cos 1_{1} \theta_{1} \ldots \sin 1_{n} \theta_{n}+\cdots\right. \\
& \equiv \sum_{1_{1}, \ldots, 1_{n}=-\infty}^{+\infty} \tilde{A}_{1_{1} \ldots 1_{n}} \exp i\left(1_{1} \theta_{1}+\cdots+1_{n} \theta_{n}\right) \text {. } \\
& \left.+A_{1_{1 \ldots 1} \ldots 1_{n}}^{s, \ldots c} \sin 1_{1} \theta_{1} \ldots \cos 1_{n} \theta_{n}+A_{1_{1 \ldots 1} \ldots 1_{n}}^{s \ldots s} \sin 1_{1} \theta_{1} \ldots \sin 1_{n} \theta_{n}\right)
\end{aligned}
$$

A general procedure for finding the symmetry groups which help to reduce the numerous terms in these expressions would involve the use of the so-called Schrödinger supergroups, which are the symmetry groups of nonrigid molecules in the Born-Oppenheimer approximation. There was an implicit application of these groups in an early paper by Wilson et al. , ${ }^{2}$ but they were first explicitly introduced by Longuet-Higgins ${ }^{3}$ and Altmann, ${ }^{4}$ who proposed two alternative general treatments; a review of the concepts and applications involved can be found in a recent article by Serre. ${ }^{5}$ Nevertheless, a more direct and intuitive approach can be more appropriate for the simple systems we shall consider in this paper; these systems are of particular interest in conformational determinations from measured magnetic parameters.

We shall mainly consider the case of a single-rotor molecule, $R-R^{\prime}$, and drop the index $i$ in $\theta$ and in the $A_{1}$ 's and $\phi_{1}$ 's. Let us assume that the group $R$ possesses symmetry $C_{n v}$ and the group $R^{\prime}$ symmetry $C_{n^{\prime} v^{\prime}}$, where the axes $C_{n}$ and $C_{n^{\prime}}$ are colinear with the torsional axis and where $v$ and $v^{\prime}=1$ or 0 depending on whether or not there are $\sigma_{v}$ planes. The parameter $A(\theta)$ will have a symmetry group $C_{m u}$ related to those of $R$ and $R^{\prime}$ and depending on the degree of specificity of $A: A$ may be an aggregate parameter such as the torsional potential $V$, dipolar moment, Raman scattering, or Zeeman splitting tensors; a mononuclear parameter such as chemical shifts $\sigma_{i}$ or hyperfine coupling tensors or constants; or a binuclear parameter such as internuclear interactions $J_{i j}$. As we shall see, the more specific the parameter $A(\theta)$, the lower its symmetry, for a given symmetry of $R$ and $R^{\prime}$.

Let us first consider the case of an aggregate param- 
eter such as $V$. The value this parameter will take depends only on the over-all conformation of $R-R^{\prime}$, not on the individual positions of its atoms. Now, for any given conformation $(\theta)$ of $R-R^{\prime}$, a superimposable conformation can be obtained by rotating, around the bond axis, either $R$ by $2 \pi / n$, or $R^{\prime}$ by $2 \pi / n^{\prime}$, or any one of the two groups by a linear combination such as $q(2 \pi / n)$ $+q^{\prime}\left(2 \pi / n^{\prime}\right)$, where $q$ and $q^{\prime} \in Z$. If $n$ and $n^{\prime}$ are both multiples of the same integer, $\epsilon$ (which, eventually, may be $n, n^{\prime}$, or 1 ), this combination can also be written $\left(q \epsilon n_{1}+q^{\prime} \epsilon n_{0}\right)\left(2 \pi / \epsilon^{2} n_{0} n_{1}\right)$. Because the expression $q n_{1}$ $+q^{\prime} n_{0}$ can now take all integer values, including those smaller than $\epsilon$, the product $\epsilon n_{0} n_{1}$, which is the lowest common multiple of $n$ and $n^{\prime}$ and will be written $n n^{\prime}$, is the order of the axis of symmetry for $V$, that is, $m=n n^{\prime}$. On the other hand, if $R$ and $R^{\prime}$ both possess one $\sigma_{v}$ plane and $\theta$ is the angle bringing $\sigma_{v^{\prime}}$ onto $\sigma_{v}$, the conformation $(-\theta)$ is equivalent to the conformation $(+\theta)$ because it is superimposable on the conformation obtained by applying the symmetry operations $\sigma_{v}$ to $R$ and $\sigma_{v^{\prime}}$ to $R^{\prime}$, as shown in Fig. 1. When $R$ and $R^{\prime}$ also possess symmetry axes, $\theta$ can be chosen as any one of the dihedral angles between pairs of $\sigma_{v}$ planes. We can thus write, very generally, $u=v v^{\prime}$. Grouping the two previous results, we write

$$
C_{m u}=C_{\underline{n n^{\prime}, v v^{\prime}}} \text {. }
$$

For a mononuclear parameter such as $\sigma_{i}$, one can no longer consider as superimposable two conformations that differ by the interchange, through either rotation or reflection, of nucleus $N_{i}$ with an equivalent nucleus on the same group. As a result, if for instance $N_{i} \in R$, then $m=n^{\prime}$ and $u=v v^{\prime}$ or 0 depending on whether or not $N_{i}$ lies on a $\sigma_{v}$ plane. We shall write this, symbolically, as

$$
C_{m u}=C_{n^{\prime}, v^{\prime} v \sigma_{i \sigma_{v}}} .
$$

For a binuclear parameter such as $J_{i j}$, one must distinguish between the two different cases where $N_{i}$ and $N_{j}$ are geminal (on $R$, say) or vicinal (on $R$ and $R^{\prime}$, respectively). The first case is similar to that of mononuclear parameters, except that now the pair $\left(N_{i}, N_{j}\right)$ must obey the symmetry $\sigma_{v}$ for a symmetry plane to exist for $J_{i j}$. We may write this as

$$
C_{m u}=C_{n^{\prime}, v^{\prime} v \delta_{\langle i, j) \sigma_{v}}} \text {. }
$$

In the second case, two conformations can be considered as superimposable only if the rotation or reflection which brings one conformation onto the other does not change the relative positions of $N_{i}$ and $N_{j}$. As a result,

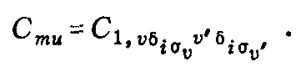

The groups defined by Eqs. (4)-(5) obviously have lower symmetries than that defined by Eq. (3). The total symmetry holding for aggregate parameters is reestablished, however, for such combinations as the effective Hamiltonian involving all $\sigma_{i}$ 's and $J_{i j}$ 's:

$$
\mathfrak{H}_{s}=\sum_{i} \hbar \gamma_{i} \mathbf{I}_{i}\left(1-\sigma_{i}\right) \mathbf{H}_{0}+\sum_{i\langle j} \sum_{j} \hbar \mathbf{I}_{i} J_{i j} \mathbf{I}_{j},
$$

provided that the distribution of nuclear moments does not have lower symmetry than the molecule $R-R^{\prime}$ itself. This situation, where the torsional angle has a defined value characteristic of the system, is not to be confused with that arising when the spin-Hamiltonian parameters are anisotropic and $\mathbf{H}_{0}$ can take all possible orientations in space. ${ }^{6}$

Once we know the symmetry group, $C_{m u}$, to which $A$ belongs, it is easy to discard the harmonics with zero coefficients in the Fourier series expansion of $A(\theta)$. First, a $C_{m}$ symmetry axis implies that, for every value of $\theta, A[\theta+p(2 \pi / m)]=A(\theta)$, where $p \in Z$. The consequence of this is that all $A_{1}^{c}$ 's and all $A_{1}^{s}$ 's in formula (1) are zero except those for which $1=k m, k$ being an integer. Second, a $\sigma_{v}$ symmetry plane (that is, $u=1$ ) implies that, for every value of $\theta$, with an appropriate choice of the origin, $A(-\theta)=A(\theta)$. The consequence of this is that all $A_{1}^{s \prime}$ 's (and, consequently, all $\phi_{1}^{\prime}$ 's) in formula (1) are zero. As an example, one may consider the $\pi$-electron free radical $\mathrm{H}_{2} \dot{\mathrm{C}}-\mathrm{CH}_{3}$, where $R \in C_{2 v}$ and $R^{r} \in C_{3 v}{ }^{7}$ According to Eqs. (3) and (4), respectively, the torsional potential $V$ possesses $C_{6 v}$ symmetry ${ }^{2}$ while the hyperfine coupling constants $a_{\beta}$ of the methyl hydrogens possess $C_{2 v}$ symmetry. ${ }^{B}$ The simplest Fourier series expansions, derived from Eq. (1), are then

$$
\begin{aligned}
& V(\theta)=V_{0}^{c}+V_{B}^{c} \cos 6 \theta \sim\left(U_{B} / 2\right)(1-\cos 6 \theta), \\
& a_{B}(\theta)=a_{0}^{c}+a_{2}^{c} \cos 2 \theta \equiv b_{0}+b_{2} \cos ^{2} \theta .
\end{aligned}
$$

If one either substitutes one or several methyl hydrogens by different atoms, or replaces the $\mathrm{H}_{2} \dot{\mathrm{C}}$ group by a bent or $\sigma$-electron radical group, one lowers the symmetries of these parameters, and this gives rise to various corrections-in particular for the hyperfine
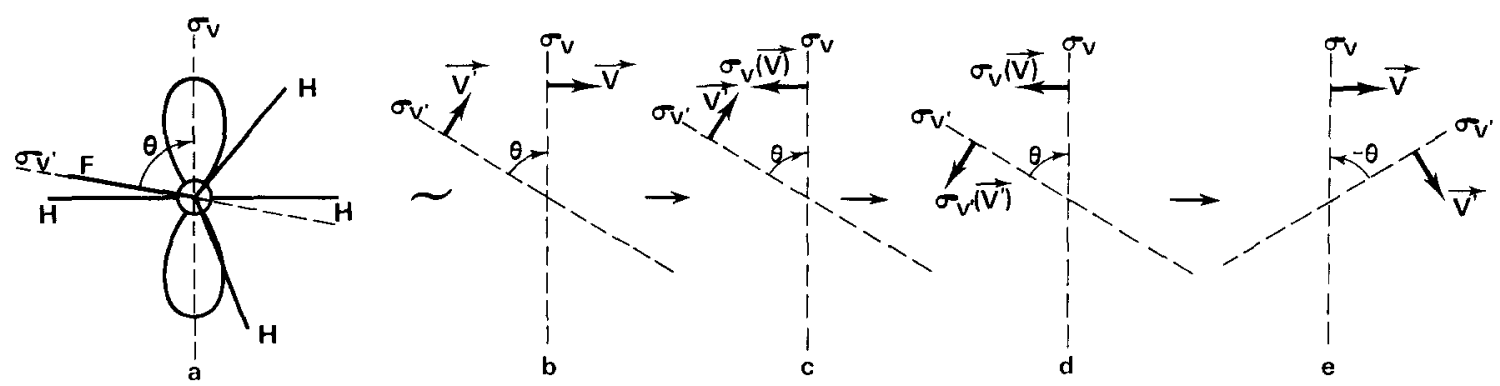

FIG. 1. Reflection symmetry of an aggregate parameter in a monorotor molecule. a: profile view of a typical molecule, $\mathrm{H}_{2} \mathrm{C}-\mathrm{CH}_{2} \mathrm{~F}$, in the conformation $(+\theta)$; $\mathrm{b}$ : schematic representation of this structure; $c$ : result obtained by reflection of the $\mathrm{H}_{2} \mathrm{C}$ group through its $\sigma_{v}$ plane; $\mathrm{d}$ : structure resulting from a consecutive reflection of $\mathrm{CH}_{2} \mathrm{~F}$ through $\sigma_{v^{\prime}}$; e: the same as b but for the conformation $(-\theta)$. Structures $d$ and e are superimposable through rotation by $180^{\circ}$ around a vertical axis. 
couplings ${ }^{2}-$ which have often been overlooked or misunderstood. ${ }^{10}$

We may now draw some general consequences from Eqs. (3) $-(5)$ for the limited expansions of the various types of parameters. Equation (3) shows that the sine terms necessarily appear for a torsional potential only when at least one of the central atoms is asymmetrically substituted $\left(v v^{\prime}=0\right)$. On the other hand, Eq. (4) shows that for chemical shifts, hyperfine couplings, etc., one may have nonzero sine terms even with no asymmetric central atom, provided that the considered parameter refers to a nucleus not lying on a $\sigma_{v}$ plane. ${ }^{9}$ Also, the order of the first cosine term is generally lower then. Equation (5b) shows that for vicinal internuclear couplings, there must always be a nonzero $\cos \theta$ term $(m=1) .{ }^{11}$ One may notice that these results are independent of the type of nuclei concerned ( $H$ or $F$, for instance), which affects only the values of the harmonic coefficients. 9,11 They stand for the different components of the representative tensor if the considered parameter is anisotropic. ${ }^{12}$ They also stand for any physically meaningful contributions in which the parameter may be split (spin delocalization and spin polarization, for instance) when these follow the symmetry of the parameter. ${ }^{13}$ They are basically independent of the quantumchemical method used to calculate the coefficients as long as the invariance requirements are respected. ${ }^{14,15}$

The extent to which some of the nonzero coefficients are numerically significant depends on the degree of departure of the considered parameter from the higher symmetries in which these coefficients are zero. The few examples given in Table I show how the departure from a given symmetry introduces a progressive increase in some initially zero coefficients. In the case of the fluorotoluene isomers, one has $R \in C_{3 v}$ and $R^{\prime} \in C_{2 v}$ if $\mathrm{F}$ is in the para position of the methyl group, $R^{\prime} \in C_{1 v}$ in the other two cases. Then, according to Eq. (3), $V \in C_{6 v}$ or $C_{3 v}$ depending on the relative positions of the substituents. However, whereas the $\cos 3 \theta$ term is only a corrective term in the meta isomer, it is the main angular-dependent term in the ortho isomer. In the case of the $\pi$-electron free radicals, $R \in C_{2 v}$ and $R^{\prime} \in C_{1 v}$ in the first two cases, and $R^{\prime} \in C_{1}$ in the last one. According to $\mathrm{Eq}$. (4), for the protons adjacent to the $\beta$ position of the radical carbon center, $a_{\beta} \in C_{2}$ in all three cases. But the $\sin 2 \theta$ term is much smaller for the cyanogen compound than with the fluorine; with the two substituents $\mathrm{CH}_{3}$ and $\mathrm{F}$, the $\sin 4 \theta$ term is no longer completely negligible (note that for the high-order harmonics the sine terms may be larger than the cosines). For the protons in the $\alpha$ position (not shown in Table I), all the angulardependent terms are much smaller than the constant. The situation should usually be similar for chemical shifts and geminal internuclear interactions, because the couplings within one rotor are not likely to be very sensitive to the orientation of the other rotor. The use of simple physical models ${ }^{7,9}$ may often help to understand the relative magnitudes of the nonzero coefficients Finally, for the $\sigma$-electron free radicals considered in the last two lines of Table $I$, up to five terms may be required to correctly represent the conformational dependence of the hyperfine couplings of the $\beta$ protons.

One may also start with a rather symmetrical structure and try to predict the form and intensity of the cor rective terms, to the simple formulas, due to some asymmetry. For instance, for the radical $\mathrm{CH}_{2}-\mathrm{CH}_{3}$, we have seen that $a_{B}=a_{0}^{c}+a_{2}^{c} \cos 2 \theta$. When one goes to the more asymmetric form $\dot{\mathrm{C}} \mathrm{H} F-\mathrm{CH}_{3}$, one has (1) loss

TABLE I. Fourier series expansion coefficients of typical parameters in monorotor molecules, $R-R^{\prime} ; 1$ refers to the harmonic order, with a prime for the sine terms. The first three lines refer to the torsional potential $V$ (in cal/mole) of the three fluorotoluene isomers: the constant term here is somewhat arbitrary and chosen so as to give a zero minimum value to $V$. The next five lines refer to the hyperfine coupling constants $a_{\beta}$ (in G per unit of spin density) of the protons adjacent to the $\beta$ position of the indicated $\pi$-el ectron (first three lines) and $\sigma$-electron (last two lines) free radicals (the four-figure numbers at the end of the lines are the conformation-averaged spin densities on the partly-filled orbital of the radical carbon center); the values are given for those protons having the least el ectrophilic substituent on the right when looking through the $R-R^{\prime}$ bond from the $R^{\prime}$ side with the proton vertically upwards: the sine terms would be opposite in sign in the reverse case. All main coefficients are underlined.

\begin{tabular}{|c|c|c|c|c|c|c|c|c|c|c|c|c|c|}
\hline$R-R^{\prime}$ & 0 & 1 & $1^{\prime}$ & 2 & $2^{\prime}$ & 3 & $3^{\prime}$ & 4 & $4^{\prime}$ & 5 & $5^{\prime}$ & 6 & $6^{\prime}$ \\
\hline para-ft. ${ }^{2}$ & 5.75 & 0 & 0 & 0 & 0 & 0 & 0 & 0 & 0 & 0 & 0 & -5.75 & 0 \\
\hline meta-ft. & $\underline{8.35}$ & 0 & 0 & 0 & 0 & $-\underline{2.75}$ & 0 & 0 & 0 & 0 & 0 & $-\underline{5.6}$ & 0 \\
\hline ortho-ft. & $\underline{33.3}$ & 0 & 0 & 0 & 0 & $\underline{29.6}$ & 0 & 0 & 0 & 0 & 0 & $-\underline{3.7}$ & 0 \\
\hline$\dot{\mathrm{C}} \mathrm{H}_{2}-\mathrm{CH}_{2}(\mathrm{CN})^{\mathrm{b}}$ & 30.78 & 0 & 0 & $\underline{28.45}$ & 0.44 & 0 & 0 & 0.13 & -0.08 & & 0.9232 & & \\
\hline$\dot{\mathrm{C}} \mathrm{H}_{2}-\mathrm{CH}_{2} \mathrm{~F}$ & $\underline{28.12}$ & 0 & 0 & $\underline{25.59}$ & $-\underline{5.99}$ & 0 & 0 & -0.04 & 0.38 & & 0.9298 & & \\
\hline$\dot{\mathrm{C}} \mathrm{H}_{2}-\mathrm{CHF}\left(\mathrm{CH}_{3}\right)$ & $\underline{28.36}$ & 0 & 0 & 26.30 & $-\underline{6.37}$ & 0 & 0 & 0.04 & $\underline{0.92}$ & & 0.9316 & & \\
\hline $\mathrm{CH}_{2}=\dot{\mathrm{C}}-\mathrm{CH}_{3}{ }^{\mathrm{b}}$ & 21.87 & -12.30 & 0 & $\underline{15.91}$ & 0 & -0.10 & 0 & 0.02 & 0 & & 0.7970 & & \\
\hline $\mathrm{O}=\dot{\mathrm{C}}-\mathrm{CH}_{2} \mathrm{~F}^{\mathrm{c}}$ & 11.17 & -20.84 & $-\underline{2.74}$ & $\underline{8.98}$ & $\underline{2.64}$ & -0.39 & -0.50 & 0.02 & 0.13 & & 0.5101 & & \\
\hline
\end{tabular}

${ }^{2}$ Calculated by CNDO. ${ }^{16}$

'Calculated by INDO.

'See, also, H. Veillard and B. Rees, Chem. Phys. Lett. 8, 267 (1971). 
of the $\mathrm{C}_{2}$ axis, because $\mathrm{H}$ and $\mathrm{F}$ are not interchangeable; (2) loss of the $\sigma_{v}$ plane, because $\dot{C} H F$ is not as planar as $\dot{\mathrm{C}} \mathrm{H}_{2} \cdot{ }^{10,17}$ As a result, both $\sin 2 \theta$ and $(\cos \theta, \sin \theta)$ terms are required. However, since the $\sin 2 \theta$ term introduced by changing a geminal $\mathrm{H}$ to an $\mathrm{F}$ is about 4 times smaller than the $\cos 2 \theta$ term, while the $\cos \theta$ term occurring in the corresponding $\sigma$-electron radical is of the same order as the $\cos 2 \theta$ term, one may guess that the $\cos \theta$ harmonic introduced by the departure from planarity will be the main correction here. In $\dot{\mathrm{C}} \mathrm{F}_{2}-\mathrm{CH}_{3}$, the latter will be the only correction. In $\dot{\mathrm{CH}}_{2}-\mathrm{CH}_{2} \mathrm{Cl}$, the configuration of the second group strongly distorts when rotated around the $\mathrm{C}-\mathrm{C}$ bond axis, ${ }^{18}$ This does not change the symmetry of any one of the two groups, however, but introduces a more complicated angular dependence of $a_{\beta}$, which can be accounted for by larger fourthorder harmonics in the limited expansion of this parameter.

\section{CONCLUSIONS}

We have shown how the symmetry properties of the constitutive groups of monorotor molecules can lead to various reductions in the Fourier series expansions of different molecular parameters such as torsional potentials, hyperfine couplings, chemical shifts, and internuclear interactions. The simple rules we have derived also apply to polyrotor molecules for the dependence of these quantities on any one of the torsional angles. When two or more torsional angles are involved, the coupling terms that appear, according to Eq. (2), can also sometimes be reduced with the help of symmetry properties. This can be done by inspection in simple cases, ${ }^{16}$ but in more complex situations use must be made of the general theory of the symmetry of nonrigid molecules. ${ }^{3,4}$ The simple considerations we have developed already provide a useful rationalization for some well-known conformational dependences which are not always clearly understood. They will be of particular interest when trying to predict or understand qualitatively the deviations from the results derived with the simple formulas in more complex situations.
* Present address: Department of Chemistry, The University of British Columbia, 2075, Wesbrook Place, Vancouver-8, E. C., Canada.

"Permanent address: Instituto de Quimica Fisica "Rocasolano," Consejo Superior de Investigaciones Cientificas, Serrano, 119, Madrid-6, Spain.

${ }^{1}$ W. J. Orville-Thomas (collective work), Internal Rotation in Molecules (Wiley, New York, 1974).

${ }^{2}$ E. B. Wilson, Jr., C. C. Lin, and D. R. Lide, Jr., J. Chem. Phys. 23, 136 (1955).

${ }^{3}$ H. C. Longuet-Higgins, Mol. Phys. 6, 445 (1963).

${ }^{4}$ S. L. Altmann, Proc. Soc. London Ser. A 298, 184 (1967); Mol. Phys. 21, 587 (1971).

${ }^{5} \mathrm{~J}$. Serre, in Advances in Guantum Chemistry (Academic, New York, 1974), Vol. 8, p. 1 .

${ }^{6} \mathrm{~J}$. Maruani, J. A. R. Coope, and C. A. MeDowell, Mol. Phys. 18, 165 (1970).

${ }^{7}$ R. W. Fessenden, J. Chim, Phys. 61, 1570 (1964).

${ }^{8}$ J. H. Freed and G. K. Fraenkel, J. Am. Chem. Soc, 86, 3477 (1964).

${ }^{9}$ D. Bahier and J. Maruani, C. R. Acad. Sci. (Paris) C 275, 257 (1972); A. Hernandez-Laguna and J. Maruani, ibid. (to be published); J. Maruani, Mol. Phys. (to be published).

${ }^{10} \mathrm{~K}$. S. Chen, P. J. Krusic, P. Meakin, and J. K. Kochi, J. Phys. Chem. 78, 2014 (1974).

${ }^{11}$ M. Karplus, J. Chem. Phys. 30, 11 (1959), G. Govil, Mol. Phys. 21, 953 (1971)

${ }^{12}$ M. Iwasaki, Mol. Phys. 20, 503 (1971)

${ }^{13} \mathrm{Y}$. Ellinger, R. Subra, B. Lévy, P. Millié, and G. Berthier, J. Chem. Phys. 62, 10 (1975); see also Localization and Delocalization in Quantum Chemistry (Reidel, Dordrecht, 1975), Vol 1, p. 283.

${ }^{14}$ F. C. Adam and F. W. King, J. Chem. Phys. 58, 2446 (1973).

${ }^{15} \mathrm{~J}$. Pople, in The World of Quantum Chemistry, Ist International Congress of Quantum Chemistry (Reidel, Dordrecht, 1974), p. 49 .

${ }^{16} \mathrm{Y}$. G. Smeyers and A. Hernandez-Laguna, in Chemical and Biochemical Reactivity, VIth Jerusalem Symposium on Quantum Chemistry and Biochemistry (Israel Academy of Sciences, Jerusalem, 1974), p. 449, A. Hernandez-Laguna, thesis, Madrid, 1974.

${ }^{17}$ R. W. Fessenden and R. H. Schuler, J. Chem. Phys, 43 2704 (1965).

${ }^{18} \mathrm{I}$. Biddles and A. Hudson, Chem. Phys. Lett. 18, 45 (1973). 